\title{
Gritt Klinkhammer* Der interreligiöse Dialog als Boundary Work
}

https://doi.org/10.1515/zfr-2019-0002

Zusammenfassung: In den folgenden Ausführungen werden die Dynamiken und Wirkungen interreligiöser Dialoggruppen beleuchtet. Es werden konstruktionstheoretische strukturelle und sozialpsychologische Perspektiven miteinander in Verbindung gebracht, um den Blick dafür zu öffnen, inwiefern der interreligiöse Dialog als eine spezifische Auseinandersetzung mit religiösen und gesellschaftlichen Grenzen zu beschreiben ist, in der die Akteure (religiöse) Identität und (religiöse) Alterität rekonfigurieren. Schließlich ist die Frage interessant, ob etwas Neues anstelle der bestehenden konfessionellen und institutionalisierten Grenzziehungen tritt. Bei dieser Betrachtung gerät auch in den Fokus, wie Religion in diesem Rahmen verhandelt und definiert wird, was schließlich die Frage aufwirft, wie sich Religionswissenschaftler`innen $\mathrm{zu}$ wandelnden konfessionellen Grenzziehungen im interreligiösen Dialog methodologisch verhalten.

Schlagwörter: Interreligiöser Dialog, Christentum, Islam, strukturale Anthropologie, sozialpsychologische Intergruppenforschung

Abstract: The author assumes that the self-declared aim of peacemaking by interreligious Grassroots-groups is accompanied by several intended as well as unintended consequences, which effect the dynamics of boundary work, by not only balancing but also creating new differentiations and conflicts between confessions, groups and identities. In order to examine in particular the construction of the distinction between identity and alterity, the following paper adopts, firstly, a structuralist perspective, and, secondly, a socio-psychological approach, both focusing on boundary work. It poses the question whether something new is replacing the existing confessional institutionalized boundaries. In doing so, this paper will also examine how religion is treated and defined within these groups and negotiations and, last but not least, how researchers in the field of the study of religion are methodically dealing with these productive dynamics of religion within interreligious dialogue.

\footnotetext{
*Kontaktperson: Dr. Gritt Klinkhammer, Universität Bremen, Fachbereich Kulturwissenschaft, Institut für Religionswissenschaft, Badgasteiner Str.1, 28359 Bremen, 
Keywords: Interreligious Dialogue, Christianity, Islam, structuralist Anthropology, social-psychological Inter-Group Research

Die Frage, die sich bei der Beobachtung von interreligiösen Dialogen stellt und die auch seitens der Politik an diese Forschung herangetragen wird, ist die, ob das Handeln dieser Gruppen nun den Frieden bzw. das friedliche Zusammenleben der Menschen mit unterschiedlichen religiösen Überzeugungen in unserer Gesellschaft befördern kann. Die Dialoggruppen selbst sind davon überzeugt, dass ihr Handeln als Beitrag zur Friedenssicherung gedeutet werden kann. Sie setzen dazu vor allem auf die Suche nach Gemeinsamkeiten und gemeinsamen Werten (vgl. "Ziele des Dialogs”, s.u.), um die scheinbar unüberbrückbaren Unterschiede zwischen den Religionen, die zum Konflikt führen oder führen können, zu überwinden. Das heißt, durch das Auffinden von Gemeinsamkeiten sollen Grenzen zwischen den Religionen überwunden werden.

Im Folgenden möchte ich darum den interreligiösen Dialog in seiner boundary work, d.h., der inkludierenden und exkludierenden Arbeit an den Grenzen zwischen den Religionen, beobachten und fragen, inwieweit hierdurch eine Annäherung aneinander und Konfliktabbau bzw. gegebenenfalls genau das Gegenteil erreicht wird.

Grundsätzlich vertritt die sozialpsychologische Vorurteilsforschung die These, dass jeglicher Kontakt zwischen unterschiedlichen Gruppen positive Effekte auf den Abbau von Vorurteilen und damit auch von stereotypen Feindbildern hat (Allport 1954, Pettigrew 2008). Im Folgenden möchte ich diese These in Bezug auf die interreligiösen Dialoge kritisch diskutieren, indem ich mithilfe der strukturalistischen Betrachtung des Geschehens als boundary work aufzeige, dass und inwiefern es sich bei den interreligiösen Dialogen um eine Arbeit an identitätsbezogenen Grenzen handelt, die dadurch weniger eine Aufhebung als vielmehr eine Festigung oder eine Verschiebung erfahren. Diese boundary work muss nicht in einen Konflikt führen - muss aber eben auch Konflikte nicht aufheben, sondern kann sie verfestigen oder auch auf andere Inhalte und Gruppen verlagern. Mithilfe der sozialpsychologischen Intergruppentheorie von Wagner (2000) sollen dann in einem zweiten Schritt weitere Bedingungen beleuchtet werden, unter denen die aufgezeigten Grenzziehungsdynamiken zu einem positiven bzw. negativen Kontakt - im Sinne der Förderung oder Verhinderung eines friedvollen Miteinanders - führen.

Schließlich legen diese Ergebnisse eine kritische Beleuchtung der vielfach bis heute vertretenen Auffassung nahe, dass Religionswissenschaft dazu prädestiniert sei, sich in den interreligiösen Dialog einzubringen. Selbstverständlich kann man sich als Religionswissenschaftler*in an interreligiösen Dialogen beteiligen wie man sich auch religiös betätigen kann; Religionswissenschaft selbst hat aber m.E. kein Potenzial, um in dieser komplexen religionsproduktiven Dynamik des interreligiösen Dialogs vermittelnd tätig zu werden. 
Im Folgenden stelle ich also zunächst einmal ausgewählte Ergebnisse aus der Dialoggruppenforschung (2011) und einer aktuell laufenden Untersuchung im gleichen Feld mit anderer Fragestellung (Klinkhammer und Neumaier 2019) vor. In einem zweiten und dritten Schritt beleuchte ich die Grenzarbeit in interreligiösen Dialogen mittels o. g. Theorien, um abschließend auf die Diskussion methodologischer Konsequenzen des Verhältnisses von Religionswissenschaft und interreligiösem Dialog einzugehen.

\section{Interreligiöse Dialoggruppen in Deutschland: Entstehung, Ziele und Aufbau}

Seit Mitte des 20. Jahrhunderts wird der interreligiöse Dialog unter dem Eindruck von Globalisierung und zunehmend religiös pluraler Gesellschaften als hervorragendes Mittel friedlicher Konvivenz verstanden. ${ }^{1}$ In Deutschland fand der institutionalisierte Dialog seinen Anfang aufgrund der Intervention der USA zwischen Juden und Christen in den 1950er Jahren und zielte in erster Linie auf Versöhnung. Mittlerweile hat allein die „Gesellschaft für jüdisch-christliche Zusammenarbeit“ mehr als 80 eigenständige Gruppen in Deutschland (Braunwarth 2011). Manche dieser Dialoge haben sich auf einen Trialog hin erweitert, indem sie den Islam als weiteren Dialogpartner hinzugezogen haben. Der christlich-muslimische Dialog setzte international nach dem sogenannten Sechstagekrieg 1967 ein (Sperber 1999) und ging in Deutschland dann auch aus der Begegnung mit den muslimischen ,Gastarbeitern“ und den ersten Debatten um eine Leitkultur Ende der 1980er Jahre hervor. Seinen bislang stärksten Aufwind erhielt er aber 2001 nach dem Terroranschlag von 9/11 und den darauf folgenden Anschlägen in Madrid und London. Die damalige Bundesregierung setzte daraufhin 2002-2006 das Programm „entimon - gegen Gewalt und Rassismus“ zum Aufbau lokaler interkultureller Bildungsprojekte ein und förderte unter diesem Motto auch lokale interreligiöse Dialoggruppen als Teil ihrer Sicherheits- und Präventions- sowie Integrationspolitik. ${ }^{2}$ Es sollten neben den Kirchen zivilgesellschaftliche Initiativen des „Dialogs der Religionen und Kulturen“ zur Integration entstehen. Die Dia-

\footnotetext{
1 Theolog^innen wie Religionswissenschaftler^innen haben sich darum über die notwendigen Bedingungen für seinen Erfolg Gedanken gemacht (Swidler 1987, 2014; Micksch 2005 u.a.). Gerne wird dazu auch auf historische Vorbilder für den interreligiösen Dialog wie das mittelalterliche Spanien hingewiesen. Allerdings dienten diese nur literarisch dokumentierten Dialoge wohl eher der Wahrheitsdurchsetzung oder der apologetischen Verteidigung des Eigenen, ohne das Verständnis eines offenen Aushandlungsprozesses mitzutragen (vgl. dazu z.B. Forst 2003).
} 
logos-Studie (Klinkhammer et al. 2011), die die Arbeit interreligiöser Dialoginitiativen unter Beteiligung von Muslim^innen in Deutschland untersucht hat, hat 2011 über 230 solcher multireligiöser Dialoginitiativen ausfindig machen können, die unabhängig und neben den offiziellen kirchlichen Dialogen agierten. Von diesen haben sich 132 Dialoginitiativen an der Befragung beteiligt. Von den befragten Gruppen bestanden $46 \%$ aus Christ*innen und Musliminnen, $44 \%$ sind multireligiöse Gruppen, $11 \%$ Trialoggruppen.

Sowohl gesellschaftspolitisch als auch in den Selbstdarstellungen der Akteure fassbar, können ganz unterschiedliche Ziele dieser Dialoge ausgemacht werden. Entsprechend dem Entstehungshintergrund einer großen Mehrheit der Dialoge aus der medialen Wahrnehmung der weltweiten kriegerisch-gewalttätigen oder terroristischen Aktivitäten islamisch-politischer Gruppen ist es fast durchgängig benanntes Ziel der Gruppen, die friedliche Koexistenz in Deutschland über den interreligiösen Dialog zu sichern. So erhalten Motive wie „gemeinsame Werte entdecken und fördern“ und „Islamfeindlichkeit und Vorurteilen entgegenzuwirken“ über 90-prozentige Zustimmung in den Gruppen (Klinkhammer et al. 2011, 46-48). Es geht dabei nicht um die Aufarbeitung der politischen Hintergründe der Gewaltausbrüche, vielmehr werden in den Gruppen in oftmals öffentlichen Diskussionsveranstaltungen Aspekte des Islams und des Christentums im Vergleich vorgestellt, die die Gemeinsamkeiten dieser beiden Religionstraditionen widerspiegeln sollen, oder es wird allgemein Aufklärungsarbeit über den Islam in öffentlichen Vorträgen und Podiumsdiskussionen betrieben (Klinkhammer et al. 2011, 68-73). Zudem haben 85 \% der Gruppen auch die Verbesserung der gemeinsamen gesellschaftlichen Lebenswelt als Ziel vor Augen und möchten mit dem Dialog das „Zusammenleben vor Ort gemeinsam gestalten“. Hier klingt bereits das von vielen Dialogteilnehmer*innen empfundene Defizit der gesellschaftlichen Partizipation und/oder Integration insbesondere von Musliminnen mit. ${ }^{3}$ Dabei betrachten drei Viertel der Gruppen den interreligiösen Dialog explizit eher unter einem Gerechtigkeits- und/oder Empowermentaspekt, indem sie damit auch die „gesellschaftliche Teilhabe von Migrant*innen fördern“ wollen.

Im engeren Sinne religionspolitisch sehen ebenfalls drei Viertel der Gruppen den interreligiösen Dialog, indem sie mit ihm grundsätzlich die „Gleichstellung aller Religionsgemeinschaften“ (76\%) fördern, d.h. hier insbesondere die Gleichstellung des Islams mit dem Christentum unterstützen wollen. Eine nicht ganz so

2 Die Integrationspolitik nahm erst etwa 2006 Fahrt auf mit der Einberufung der jährlichen Integrationsgipfel der Bundeskanzlerin Merkel sowie der Deutschen Islamkonferenz (DIK) durch den damaligen Innenminister Schäuble.

3 Zusätzlich zu der Gruppenbefragung haben sich 298 Mitglieder aus 57 Gruppen an einer Teilnehmer^innenbefragung durch die Dialogos-Studie beteiligt. 
breite, aber doch auch signifikante Zustimmung hat demgegenüber das Ziel erhalten, in einem solchen interreligiösen Dialog die „Stärkung religiöser Interessen gegenüber dem Staat“ (32\%) voranzutreiben. In dieser Aussage macht sich zudem eine säkularisierungskritische Haltung bemerkbar.

In eine noch ganz andere Richtung geht die breite Zustimmung der befragten Gruppen zum Ziel, „die eigene Religion besser zu verstehen“ (81\%): Hier geht es um die Möglichkeit, in den Begegnungen mit den anderen die eigene Religion zu ergründen und neu über sie nachzudenken. Die Dialoggruppe scheint das Nachdenken und Gespräch über das Eigene besonders anzuregen. Ein fortgeschrittenes Selbstverständnis von Individualisierung und Selbstermächtigung in Bezug auf Religion scheint sich hierin ebenfalls auszudrücken. Dies stimmt auch überein mit der Offenheit der Gruppen für unterschiedliche Ziele der Teilnehmer`innen.

Die Dialogos-Studie hat interreligiöse Dialoginitiativen (unter Beteiligung von Muslim^innen) untersucht, die sich eigens zum Zweck des Dialogs gebildet haben. ${ }^{4}$ Von diesen eigenständigen Dialoggruppen haben sich immerhin etwa $20 \%$ sogar als eingetragener Verein organisiert. Eine Zielgruppenkonzentration ist bei den wenigsten Dialoggruppen ausgeprägt; die meisten Gruppen verstehen sich als für alle Interessierten offen - also für Alte/Junge, Männer/Frauen, religiöse Amtsträger oder Theolog^innen sowie Laien.

Drei Viertel der Initiativen bringen regelmäßig zwischen 11 und 35 Personen zusammen. Die Fluktuation ist dabei relativ gering. Insgesamt verzeichnen die Gruppen nach eigenen Angaben wachsende Teilnehmer^innenzahlen. Allerdings beklagten $20 \%$ der Initiativen eine Rückläufigkeit der Teilnahme bzw. Fluktuation unter den Musliminnen.

Insgesamt ist der Altersdurchschnitt der Teilnehmer*innen relativ hoch: $67 \%$ sind zwischen 30 und 50 Jahre alt. Bei immerhin $30 \%$ bilden die über 50 Jährigen die größte Gruppe. Die beteiligten Musliminnen sind dabei tendenziell jünger als die Christ^innen. Der Bildungsstand innerhalb der Gruppen ist zudem überdurchschnittlich hoch. Rund $80 \%$ verfügen über eine allgemeine oder fachgebundene Hochschulreife. ${ }^{5}$ Sicherlich ist das auch einer Arbeitsform geschuldet, die vor allem auf das Gespräch setzt, obwohl auch Besuche von Gebets- bzw.

\footnotetext{
4 Darüber hinaus wurden (jenseits der ca. 230 Initiativen) Akademieprojekte, offizielle Dialoge von Kirchen-/Verbandsleitungen und Kirchengemeindeinitiativen identifiziert, die aber in der Dialogos-Studie keine Berücksichtigung gefunden haben.

5 Unter denjenigen, die den Teilnehmer^innenfragebogen ausgefüllt haben, ist allerdings eine überdurchschnittliche Beteiligung von Mittelschichtsangehörigen zu vermuten; insgesamt liegt der Bildungsdurchschnitt wahrscheinlich etwas tiefer, wie auch unsere teilnehmende Beobachtung in ausgewählten Initiativen vermuten lässt.
} 
Gotteshäusern unternommen, Meditationen und gemeinsame Gebete durchgeführt sowie Informationsveranstaltungen (Vortragsveranstaltungen) oder gemeinsame Projekte vor Ort (Feste, Aktionstage, Friedensgebete u. ä.) geplant werden.

Thematisch sind die Dialoginitiativen ebenfalls recht breit aufgestellt: Fast alle Initiativen geben an, sowohl religiös-spirituelle und theologische als auch gesellschafts- und integrationspolitisch ausgerichtete Themen zu behandeln. Bei fast drei Viertel der Gruppen spielen integrationspolitische Fragestellungen eine wichtige Rolle. Die Bearbeitung bzw. Lösung konkreter Interessenskonflikte nimmt in der Arbeit der Dialoginitiativen den geringsten Stellenwert ein. ${ }^{6}$

Bezüglich der Verteilung der Geschlechter ist auffällig, dass trotz Hinzuziehung von zehn reinen Fraueninitiativen insgesamt der Anteil der Frauen deutlich unter dem der Männer liegt. Initiativen, in denen Frauen die Mehrheit bilden, sind seltener anzutreffen (20\%). Diese Ungleichverteilung gilt für die muslimischen und die christlichen Beteiligten gleichermaßen.

Trotz des auch konflikthaften Charakters vieler Treffen weisen die Initiativen eine recht hohe Kontinuität unter den Teilnehmenden auf: Über $70 \%$ der Initiativen setzen sich mehrheitlich aus Personen zusammen, die sich bereits mehr als zwei Jahre in der Initiative engagieren (Klinkhammer et al. 2011, 51f.). Darüber hinaus hat sich gezeigt, dass die Teilnehmenden - verglichen mit dem bundesdeutschen Durchschnitt (Religionsmonitor 2008) - ihre Religiosität überdurchschnittlich stark reflektieren (Klinkhammer et al. 2011, 136f.). Das heißt, die Dialoginitiativen stellen über einen längeren Zeitraum einen stabilen Identifikationsrahmen für die Teilnehmenden dar. ${ }^{7}$

Gut $40 \%$ der nicht-muslimischen Teilnehmer*innen hatten, bevor sie Mitglied einer Dialoggruppe wurden, kaum oder keinen Kontakt mit Muslim^innen (Klinkhammer et al. 2011, 144). Dialoginitiativen sind also zu weiten Teilen auch interkulturelle und interreligiöse Kontaktbörsen, die die Pluralitätserfahrung im sozialen Nahbereich herstellen, die gesamtgesellschaftlich und medial bereits als normal gilt. Das mag auch erklären, warum viele dieser Gruppen - auch wenn sie

\footnotetext{
$6 \mathrm{Zu}$ dieser geringen Bearbeitung konkreter Konflikte passt, dass nur etwa die Hälfte der Dialoggruppen als Grund ihrer Gründung nachbarschaftliches Interesse angeben, letztlich aber die Gründungsdaten sowie die Aussage der anderen Hälfte der Gruppen darauf hinweisen, dass es v.a. die (weltweiten) politischen Ereignisse sind, die zur Gründung von Dialoggruppen geführt haben. Die lokale Situation bzw. Probleme vor Ort wurden als Grund nur vereinzelt genannt (vgl. Klinkhammer et al. 2011, 39-45).

7 Inwiefern die Dialoggruppen in religiöser Hinsicht damit auch identitätsprägend sind, bearbeitet ein laufendes Forschungsprojekt zum Thema „Identität angesichts religiöser Pluralität“, das von Anna Neumaier zusammen mit der Autorin bearbeitet und von der DFG (2014-2018) gefördert wird.
} 
auf lokaler Ebene gegründet sind - sich an Themen und Debatten zum Islam aus den Medien orientieren. ${ }^{8}$

Insgesamt lässt sich zudem aus den Ergebnissen der Dialogos-Studie ableiten, dass es sich bei einem Großteil der interreligiösen Dialoggruppen um die Etablierung einer eigenen Kultur der interreligiösen Begegnung handelt. Es zeigte sich zum einen, dass die meisten der Dialoginitiativen autonom und relativ individuell arbeiten und kaum an offizielle Dialoge ihrer Amtskirchen, islamischen Verbände $u$. ä. rückgebunden sind bzw. dem Diskurs ihrer Amtskirchen und Verbände kritisch gegenüberstehen. Sogenannte Stellvertreterdialoge werden in den wenigsten dieser Gruppen geführt - zumeist geht es um einen möglichst direkten und „authentischen“ Austausch. Zum anderen werden in vielen Initiativen sowohl eigens arrangierte Themen des Vergleichs in den Mittelpunkt gestellt („Abrahamitische Religionen“, „monotheistische Religionen“, „Bewahrung der Schöpfung “ u.a.) wie auch neue Orte (Rathäuser, Bürgerhäuser, öffentliche Plätze u.a.) für die jeweiligen Ziele in Anspruch genommen und neue gemeinsame religiöse Rituale (Eingangsgesänge, Bittgebete, Handkreise mit Begrüßung u.a.) kreiert.

Werden also in den interreligiösen Dialogen die herrschenden Grenzen zwischen den Religionsgemeinschaften aufgehoben und durch eine neue Einheit ersetzt? Lassen sich in derartigen Kontexten überhaupt noch sinnvoll Religionsgrenzen identifizieren? Oder werden die bestehenden Grenzen zwischen den Religionsgemeinschaften und auch zwischen den Religionen und der säkularen Gesellschaft neu verhandelt? Und welche Art von Grenzen wird dann neu gezogen? Führt dies alles zu einer friedlichen Koexistenz, zur Verschärfung der Grenzen oder nur zu einer neuen ausdifferenzierten Gemeinschaft der Interreligiösen? Die Frage nach den Grenzziehungsdynamiken im interreligiösen Dialog soll im Folgenden anhand theoretischer Überlegungen zur boundary work aus der strukturalen Anthropologie und der Sozialpsychologie sowie unter Berücksichtigung der Ergebnisse der Dialogos-Studie erörtert werden.

8 Dass dies oftmals zu einer sehr ambivalenten Reaktion seitens der beteiligten Muslim^innen führt, lässt sich nachvollziehen, da dann oftmals das medial fokussierte Extrem von Islam sowie globale Konflikte als Hintergrundfolie bzw. Thema für die lokale (oftmals erste und/oder einzige) Begegnung zwischen Musliminnen und Christinnen dienen. 


\section{Grenzmarkierung und Grenzüberschreitung im Interreligiösen Dialog}

Zur Frage, wozu ein Dialog gut ist, liegen verschiedene prominente philosophische Reflexionen vor, die deutlich machen, dass ein Dialog nicht nur als Gespräch zwischen zwei Parteien verstanden wird, sondern darüber hinaus sowohl ein grundlegendes Instrument der Erkenntnisgewinnung als auch der Selbstfindung darstellt. So hat Platon den Dialog als philosophische Darstellungsform gewählt, um philosophische Wahrheitsprobleme zu diskutieren und ihre Aporien sichtbar zu machen. Der Philosoph Hans-Georg Gadamer (1960) bestimmte darüber hinaus die dialogische Situation als eine zentrale Möglichkeit zur Selbsterkenntnis, indem er den Dialog als fortschreitenden reflexiven Lernprozess über sich selbst darstellte. Und der jüdische Gelehrte und Religionsphilosoph Martin Buber sah im Dialog sogar ganz grundsätzlich die Bedingung zur Selbstwerdung: „Der Mensch wird am Du zum Ich“ heißt es in seinem poetisch-philosophischen Werk „Ich und Du“ (1923, 37).

Es lässt sich unschwer der implizite Nexus der Teilnehmer^innen am interreligiösen Dialog zu solchen philosophischen Ideen erkennen, wenn sich beispielsweise mehr als 80\% der Befragten durch den Dialog mit anderen Religionsverstreter^innen auch die Verbesserung des Verständnisses der eigenen Religion erhoffen. Insgesamt zielt eine solche philosophische Betrachtungsweise des Dialogs als Selbsterkenntnis zunächst nicht unbedingt gleichzeitig auf das Ziel gesellschaftlicher Problemlösung: Andere und sich selbst $\mathrm{zu}$ verstehen, heißt noch nicht, eine Lösung im Dissens gefunden zu haben, sondern kann allenfalls ein (wichtiger) Schritt dahin sein. Dennoch hatte nicht nur die Bundesregierung interreligiöse Dialoge als ein Problemlösungsinstrument eingesetzt, als sie 2006 mit einem eigens aufgelegten Förderprogramm zur Rassismusprävention und Intervention in Dialoginitiativen investierte, auch der Europarat hat 2008 in seinem „White Paper on Intercultural Dialogue“ in Bezug auf den interreligiösen Dialog das Ziel formuliert: „Interreligious dialogue can also contribute to a stronger consensus within society regarding the solution to social problems“ (Council of Europe 2008, 24).

Diskutiert man die Arbeitsweisen von interreligiösen Dialogen im Rahmen von kultur- und sozialwissenschaftlichen sowie sozialpsychologischen Theorien über Gruppenbildungs-, Identifikations- und Alteritätsprozesse, geraten neben den oben erwähnten normativ-ethischen auch konstruktionstheoretische, strukturelle und psychosoziale Identitätsbildungsdynamiken der Austausch- und Konfrontationsprozesse in den Blick. Grundsätzlich ist hier zum ersten auf die frühen sozialkonstruktivistischen Betrachtungen des Ethnologen Fredrik Barth (1969) 
hinzuweisen, ${ }^{9}$ in denen er sich der Entstehung und Konstruktion von ethnischen Identitäten über Grenzbildungsprozesse im Kontakt widmet. Ethnische Identitäten seien mitnichten als gegeben zu betrachten und an territoriale Grenzen gebunden, sondern bildeten sich danach überhaupt erst zur Strukturierung und Organisation von Interaktion heraus ${ }^{10}$ und seien überdies veränderbar. Da im Kontext dieses Artikels vor allem religiöse Gruppenbildungen in den Blick genommen werden, möchte ich an dieser Stelle die Frage der Bildung ethnischer Identitäten nicht weiter vertiefen. Unmittelbar einsichtig im Anschluss an das Gesagte ist, dass religiöse Gruppenbildungen von sozialen und historischen Kontexten und Interaktionsprozessen geleitet und geprägt sind. Aus dieser konstruktivistischen Betrachtungsweise ergibt sich sodann auch die These, dass Kontakt und Austausch zwischen Kollektiven zur Grenzziehung anregt. So folgert Barth:

„[...] categorical ethnic distinctions do not depend on an absence of mobility, contact and information, but do entail social processes of exclusion and incorporation whereby discrete categories are maintained despite changing anticipation and membership in the course of individual life history“ (Barth 1969, 9).

Auch religionsgeschichtlich ist bekannt, dass Religionsgemeinschaften besonders dann ihr eigenes Profil geschärft haben, wenn ihnen konkrete andere religiöse Kollektive zum Gegenüber wurden. ${ }^{11}$

\subsection{Eine strukturalistische Grammatik der Boundary Work}

Der Amsterdamer Sozialanthropologe Gerd Baumann stimmt zwar in der Beobachtung der sozialen Konstruktion von Gruppengrenzen in Kontaktsituationen mit Barth überein, konstatiert allerdings darüber hinaus, dass für die soziale Konstruktion von Gruppengrenzen letztlich eine konkrete strukturelle Begrenztheit von Grammatiken des Zusammenspiels von Identität und Alterität identifizierbar sei. In seinem diesen Ansatz fundierenden Aufsatz „Grammars of Identity/Alteri-

9 Ein Dank gilt hier Paula Schrode, die mich mit ihrer Idee dieses Sonderbandes auf diese bereits frühe Beschäftigung mit der sozialen Konstruktion von Gemeinschaft in der Kulturwissenschaft hingewiesen hat.

10 So dass eine klare Zuordnung und Unterscheidung von Gruppe „X“ und Gruppe „Y“ und Gruppe „Z“" möglich wird.

11 So das Judentum an der Entstehung des Christentums wie der Katholizismus an der Entstehung des Protestantismus oder der Protestantismus auch an der Begegnung mit dem Islam. 
ty: A Structural Approach“ (Baumann 2004) unterscheidet er drei Grammatiken bzw. „Klassifikationsstrukturen“ (Baumann 2004, 19) von Inklusion und Exklusion, die für die Identitätsbildung - auf individueller wie auf kollektiver Ebene von Bedeutung sind. Aufbauend auf der basalen dichotomen Struktur von „we are good, and they are bad“ (Baumann 2004) und anschließend an die Arbeiten von Edward E. Evans-Pritchard, Edward Said und Louis Dumont arbeitet Baumann drei darauf aufbauende Gebilde des Zusammenspiels von Identität und Alterität aus, die er letztlich als nicht mehr nur binären, sondern ternären strukturellen Rahmen von Grenzziehungsprozessen versteht.

Zum Ersten finde man die Grammatik der Orientalisierung vor, die nicht nur binär klassifiziere, sondern vor allem als eine Art Umkehrungs-Spiegelbild und damit zweischneidig funktioniere:

„[...] it seems to be implicit in Said's recognition that Westerners not only denigrated that which they called ,oriental', but also desired it. [...] What made the caricatures intellectually interesting and aesthetically challenging for such elites, many of them tired of and estranged from their own cultural milieus, was also the cultural self-critique that an orientalizing of the other made possible and, as importantly, communicable“ (Baumann 2004, 20).

Die Grundgrammatik von „us = good“ und „them = bad“ werde also in Form eines dialektischen Verhältnisses zum Anderen doppelt und subtil gespiegelt. Die negative Spiegelung des Anderen (z.B. rational vs. irrational, aufgeklärt vs. abergläubisch) erhält mehr oder weniger implizit auch ein „positives“ othering (z.B. berechnend vs. spontan, materialistisch vs. mystisch). Die Orientalisierung von Religion ist religionsgeschichtlich in den letzten Jahren intensiver aufgearbeitet worden. Auch dabei wurde gezeigt, dass historisch ein starker Antrieb nicht allein die Missionierung und Unterordnung des Fremden (vgl. Said 1978) war, sondern bisweilen auch die Suche nach Vervollständigung der eigenen religiösen Kultur bzw. Identität (vgl. z.B. Brunotte 2009 oder Klinkhammer 2009).

Zum Zweiten unterscheidet Baumann die Grammatik der Segmentierung. Dieses Modell zeichnet sich durch Identifikation und Othering, durch Prozesse der Spaltung und Fusion aus. Das Besondere der Segmentierungsgrammatik sei, dass sie sich kontextuell ändert: Was in dem einen Kontext Identifizierung mit dem Anderen bedeute, könne in dem anderen Kontext Abspaltung von dem Anderen bedeuten. Für Baumann steht dazu Evans-Pritchards Darstellung der kontextabhängigen Bedeutung bei den Nuer Pate: „Who I am at what moment, is a question of context; and defining the context is a question of knowing the right, contextually appropriate, classificatory level“ (Baumann 2004, 24). Segmentierende Identifikation und Othering ist z.B. auf der Ebene der ortsgebundenen Identifizierung eingängig: Ob ich mich als Hesse, Deutscher, Europäer oder Weltbürger bezeichne, hängt sehr vom Kontext ab, in dem ich mich gerade bewege; ebenso, ob 
sich jemand als Gläubiger, Muslim, Sunnit oder Naqshbandi bezeichnet. „The crucial question, however, is [...]: who is to have the power to define the ultimate apex of the pyramid, and who defines which intervening classificatory levels?“ (Baumann 2004).

Zum Dritten ist die Grammatik der Einschließung („Encompassment“) beobachtbar. Einschließung meint einen Prozess der Anpassung und Kooptierung an das Eigene: „You may think that you differ from me in your sense of values or identity; but deep down, or rather higher up, you are but a part of me“ (Baumann 2004, 25). Diese Grammatik der Einschließung sei sehr flexibel, aber seitens ihrer Vertreter immer hierarchisierend und dominierend eingesetzt. So würden beispielsweise aus der Perspektive der meisten „Southall Hindus“ (in London) die Konflikte in Indien um Differenzansprüche der Sikhs als unnötig abgetan: Schließlich seien Sikhs letztlich Hindus wie Christen eben auch. Die Differenzziehungen vonseiten der Minderheiten würden also in dieser Grammatik der Einschließung als eine Fiktion von Identitätspolitik abgetan und alle Minderheiten letztlich als untergeordnet unter ein „encompassing Us“ subsumiert (Baumann 2004). ${ }^{12}$

\begin{tabular}{|c|c|c|c|}
\hline $\begin{array}{l}\text { Orient } \\
\text { Self }+\end{array}$ & $\begin{array}{l}\text { alism: } \\
\text { Other - }\end{array}$ & $\begin{array}{c}\text { Segmentation: } \\
\wedge \\
\wedge\end{array}$ & $\begin{array}{l}\text { Encompassment: } \\
\text { Self as Whole } \\
\wedge\end{array}$ \\
\hline Self - & Other + & $\begin{array}{l}ハ \wedge \\
\wedge \wedge \wedge \wedge\end{array}$ & $\begin{array}{l}\text { Self } \leftarrow \begin{array}{l}\text { Other as } \\
\text { as Part }\end{array} \text { Sub-Part }\end{array}$ \\
\hline
\end{tabular}

Grafik 1: Gerd Baumann 2004, 27.

Alle drei Grammatiken sind unschwer im interreligiösen Dialog erkennbar. Entsprechend der Ziele des interreligiösen Dialogs, die gemeinsamen Werte in den Vordergrund zu stellen, spielt die segmentäre Grammatik im interreligiösen Dialog eine zentrale Rolle. Bekanntes Beispiel für die gemeinsame Spitze der segmentären Pyramide im christlich-islamischen Dialog oder im jüdisch-christlich-isla-

12 Die Figur der Einschließung kommt zudem dem Modell des Inklusivismus der „Theologie der Religionen" nahe, in dem anderen Religionen auch Heilsbedeutsamkeit zugesprochen wird, aber nur insofern als sie letztlich in der wahren einen Religion als implizit inkludiert verstanden sind. Die katholische Kirche deutet ebenfalls die anderen Religionen unter Bezug auf ein solches Modell, insofern sie betont, dass auch nicht-christliche Gläubige unsichtbar an der Gnade Jesu Christi teilhaben und somit in die eine Heilige Kirche eingegliedert sind (Dominus Jesus, 2000). 
mischen Trialog ist die Idee der ,abrahamitischen Religionen“. Sofern der interreligiöse Dialog nicht nur trialogisch sondern multireligiös ausgerichtet ist, steht hierfür die Idee der „Menschen im Glauben“ oder auch die Küng’sche Religionstheologie eines gemeinsamen „Weltethos der Religionen“. Eine solche segmentäre Ausrichtung wird unterstützt durch weitere identitätsbildende thematische Vertiefungen in den Dialogsitzungen etwa über die „gemeinsame Sorge um die Schöpfung“ oder den verbindenden „Monotheismus“ und ähnliches. Eine positive orientalisierende Grammatik, die in dem Anderen jeweils immer auch Eigenschaften vermutet, die für die eigene religiöse Identität als von Vorteil oder als verloren gelten, kann bisweilen die segmentäre Grammatik unterstützen. Solch eine Strukturierung ist $\mathrm{zu}$ beobachten, wenn sich evangelische Christ*innen im Dialog beispielsweise spirituellen Traditionen des Sufismus oder sunnitische Muslim^innen sich der kritischen Textexegese annähern, weil sie darin je ein Defizit ihrer eigenen Religionstradition vermuten.

Problematisch wird die Etablierung einer segmentären Grammatik allerdings dort, wo unterhalb der gemeinsamen Spitze eine negative orientalisierende Grammatik oder die einschließende als eine vereinnahmende Grammatik vorherrscht. So ist zum einen $\mathrm{zu}$ beobachten, dass dort, wo orientalisierende Projekte wie das der „Zivilisierung des Islam“ im Dialog vorangetrieben werden, die Dialogpartner kaum gemeinsame religiöse Erzählungen finden. Der Islam bildet dabei vor allem eine Negativfolie, dessen positive Seite erst in seiner Umgestaltung sichtbar und möglich würde: Abschaffung der Scharia oder Aufbau einer geordneten islamischen Institution, Befreiung der Frau oder Aufklärung in Bezug auf den Koran sind dabei typische in solchen Gruppen verhandelte Themen. Der Grenzdiskurs verläuft dann innerhalb der Dialoggruppe und ist hier eher von Exklusion als von Inklusion geprägt. Eine vereinnahmende Grammatik ist dort zu beobachten, wo beispielsweise Muslim^innen die christlichen und jüdischen Anderen vornehmlich als „Leute des Buches“ (ahl al-kitāb) verstehen, die laut Koran ihre Heiligen Schriften jeweils verfälscht hätten und somit letztlich noch zum Islam bekehrt werden müssten. Eine vereinnahmende Grammatik der Grenzziehung kann auch als grundsätzliches Bekehrungsansinnen auftreten: Die Anderen - egal welcher Konfession - werden dann vor allem als die noch zu Bekehrenden wahrgenommen. Letztere Attitüde wird oftmals von außen, seitens der nichtteilnehmenden Glaubensgeschwister, dem Dialoggeschehen grundsätzlich kritisch unterstellt (wie Dialogteilnehmer*innen immer wieder berichten), trifft aber doch selten für die Dialoggruppen zu. Vielmehr ist zu beobachten, dass langjährige Teilnehmer*innen auf beiden Seiten sich soweit mit dem neuen gemeinsamen Grenzdiskurs identifizieren, dass der konventionelle Diskurs, der rituelle und dogmatische Grenzen zwischen den institutionalisierten Konfessionen von Christentum und Islam setzt, kaum mehr Geltung für die Religiosität der Teilnehmer^innen besitzt. 
Religiosität zeigt sich hier individualisiert und oftmals institutionen- und konfessionskritisch. ${ }^{13}$

Es zeigt sich aber auch in der Dialogos-Studie, dass sich die Grammatik der Dialoggruppen jederzeit verändern kann, je nachdem, welche Personen daran teilnehmen und welche politischen, medial kommunizierten Ereignisse das Geschehen aktuell beeinflussen. Gerd Baumann betont ebenfalls für das Verständnis seiner Grammatiken:

„The grammars provide a repertoire of structures through which to put forward arguments about self and other; but it is crucial to stress that all grammars are always at the disposal of all social actors, and it is precisely the constantly shifting invocations and revocations of each grammar that matter in the social processes of selfing and othering as we can observe empirically“ (Baumann 2004, 31).

\section{Die Ternarität der Grenzziehung}

Nun zieht Baumann eine weitere Differenzierung ein, indem er die bislang behandelte Binarität der Beispiele aus der Beobachterperspektive letztlich als Ternarität entlarvt. So werde in der segmentären Grammatik sowie der Grammatik der Einschließung nicht nur ein „Wir“ (we/us) und ein „Ihr“ (you) als die Anderen und das Gegenüber der Auseinandersetzung unterschieden, sondern ein drittes Kollektiv (them) bestimmt, das gänzlich exkludiert wird und nicht an der Auseinandersetzung oder am Dialog partizipiert und somit weder zum „Wir“(we/us) noch zum „Ihr“(you) gehört. Und in der Tat zeigen sich im interreligiösen Dialog Abgrenzungen, die indirekt oder direkt auch auf Exklusion hinweisen, die es zu reflektieren gilt, um den Kontext des Dialoggeschehen vollständig zu erfassen und die Grenzziehungsdynamiken verstehen zu können. Im interreligiösen Dialog und von den gesellschaftlichen Förderern des Dialogs werden Dritte beispielsweise als „Fundamentalisten“ oder „Intolerante“ (Religiöse) identifiziert bzw. ausgeschlossen. ${ }^{14}$ Dialogteilnahme wird hier prinzipiell als Akt der Toleranz verstanden und die Teilnahme entsprechend anerkannt. Für sogenannte praktizierende Muslim^innen, denen sowieso oftmals in Deutschland grundsätzlich eine fundamentalistische Haltung unterstellt wird, bedeutet dies entsprechend, dass die Mitgliedschaft in einer interreligiösen Gruppe - egal mit welchem persönlichen Engagement oder welcher Überzeugung -

13 Auch über diesen Zusammenhang von inter- bzw. transreligiöser und konfessioneller Identifikation versucht das bereits angeführte laufende Projekt von Klinkhammer und Neumaier Aufschluss zu erhalten; vgl. dies. (im Druck) und mehr hierzu auch in Kap. 3.

14 Vgl. hierzu auch die Diskussion über das Zulassen der Beteiligung der „Islamischen Gemeinschaft Millî Görüş e. V.“ (IGMG) u. ä. Gemeinschaften am Dialog (s. Klinkhammer u.a. 2011, 1-2.) 
als Erweis einer besonders offenen Religiosität gilt. Aber auch für Christ^innen scheint bisweilen allein die Teilnahme am Dialog schon auszureichen, um die eigene Haltung als tolerant zu bezeichnen, auch wenn sich ihre ablehnende Haltung gegenüber dem Islam im Allgemeinen (ausgenommen einzelne muslimische Dialogteilnehmer*innen) nicht geändert hat.

Überdies kann im Dialog der Ausschluss des Dritten dasjenige Kollektiv bezeichnen, das grundsätzlich nicht zu dem im interreligiösen Dialog verhandelten Grundkonflikt zu gehören scheint: die Nicht-Religiösen. Deutlich wird die Grenzmarkierung hierzu insbesondere dann, wenn sich die interreligiöse Dialoggruppe in einer gemeinsamen religiös-spirituellen Haltung (,Menschen im Glauben“) zusammenfindet (als Spitze der Segmentär-Grammatik). Solche Gruppen bilden teilweise eigene religiöse Rituale aus, die sie bewusst auf die säkulare Öffentlichkeit hin anlegen (z.B. Friedensgebete auf öffentlichen Plätzen oder in Rathäusern). Dieser Dialog zielt dann latent auch darauf, die säkularisierte Öffentlichkeit auf die (zu wenig beachtete) Bedeutung von Religion und ihren Beitrag zum Frieden aufmerksam zu machen. Das heißt, es geht hier weniger darum, die ausgeschlossenen Dritten in den Dialog hinein zu holen, als vielmehr deren Exklusion und Defizit zu markieren.

Die orientalistische Grammatik demgegenüber zeichnet sich nach Baumann durch eine zum Teil zeitlich gestaffelte Dreiteiligkeit aus. Während zunächst nur die Unterscheidung zwischen dem Eigenen (self) und dem Anderen (other) von Bedeutung ist, verändert sich die Situation der Orientalisierung im Verhältnis der migrierten Anderen der ersten Phase (old-others), die nun in Teilen integriert oder teilweise assimiliert sind und den Neuankömmlingen (new-others). Dabei könne es dazu kommen, dass zunächst das mehrheitliche kollektive „Wir“ (self) die „alten“ und die „neuen Anderen“ gegeneinander setzt und eventuell gegeneinander ausspielt, mit der Zeit aber auch die alten Anderen in das allgemeine „Wir“ (self) einreiht. Im Zuge der Integrationsdebatten in Deutschland lässt sich diese Dynamik an der einseitigen Fokussierung auf Fremdreligiöse bzw. Muslim^innen ablesen, während andere soziale Ausschlussmechanismen auf der Basis von Behinderung, Alter, Klasse und Einkommen kaum mehr öffentliche Beachtung finden. Spätestens aber seit der jüngsten Flüchtlingsdebatte werden von unterschiedlichen Seiten orientalisierende Grenzdiskurse eingezogen - seien es die old-migrants selbst, die zum „Wir“ gerechnet werden möchten, oder auch das kollektive „Wir“, das nun auf die Zugehörigkeit und Solidarität der old-migrants gegenüber der Gesamtgesellschaft angesichts der neuen Krise abstellt. ${ }^{15}$

15 Ein ganz anderes Beispiel der dreigliedrigen orientalisierenden Grenzmarkierung zeigt sich bei den Zeugen Jehovas, die sich mit der Anerkennung der Körperschaftsrechte gegen eine Abwertung 


\subsection{Grenzkonflikte und sozialpsychologische Lösungsansätze}

Es springt unmittelbar ins Auge, dass solche Identifizierungs- und Alterisierungsprozesse nicht immer konfliktfrei ablaufen, und es fragt sich, welche Bedingungen diese Konflikte in Gang setzen und unter welchen Bedingungen sie gelöst werden können. Das kann die dargelegte strukturale Betrachtungsweise selbst nicht beantworten, weshalb ich an dieser Stelle auf sozialpsychologische Ansätze zurückgreife. Aus sozialpsychologischer Perspektive zeigt sich in dem Verhalten der Wir-Gruppenbildung und -abgrenzung zunächst einmal ein quasi natürliches Identitätsbildungsverhalten des Menschen. Menschen tendieren in ihrem Verhalten dazu, sich über Gruppenbildung (d.h. auch über Grenzmarkierung bzw. Exklusion Anderer) zu identifizieren und generell die eigene Gruppe gegenüber der Umwelt oder einem anderen Kollektiv zu unterscheiden. Dazu nutzen die Gruppenmitglieder Kategorien der Auf- oder Abwertung, wobei die Eigengruppe aufund die Fremdgruppe abgewertet werde. ${ }^{16}$

Vor dem Hintergrund solcher sozialpsychologischer Mechanismen der wertenden Grenzmarkierung von Gruppen erscheint es höchst plausibel, dass religiös-plurale Gesellschaften fortwährend mit Deutungs- und Anerkennungskämpfen unter ihren Mitgliedern bzw. Gruppen zu tun haben. Gerd Baumann sieht dabei nicht in den aufgezeigten Grammatiken der Grenzziehung selbst, sondern erst in ihrer Verhärtung und Reduzierung auf die Nichtanerkennung des Anderen, und damit die Nichtanerkennung von Differenz, den Auslöser für den Ausbruch von Gewalt. Das heißt, Baumann sieht letztlich in der Grenzmarkierung und ihrer Verhandlung zunächst keine Dysfunktionalität für die Gesellschaft. Die Sozialpsychologie kategorisiert ähnliche Grundmuster der Grenzbeziehung wie Baumann und geht ebenfalls davon aus, dass Konfliktlösungen nicht durch die Auflösung von Grenzen, sondern vielmehr über die flexible Verschiebung von Gruppengrenzen erreicht werden. Grundsätzlich geht die Sozialpsychologie zunächst von der „mere contact hypothesis“ (Allport 1954, Pettigrew 2008 u.a.) aus, die besagt, dass sich im Prinzip jeder Kontakt zwischen Personen unterschiedlicher sozialer

als „Sekte“ stellen möchten: Sekten bleiben dann die Anderen. Allerdings zeigt sich an diesem Beispiel auch, dass Deutungshoheiten durch solche formalen Akte nicht gebrochen werden können; rechtlich sind die ZJ zwar als Religionsgemeinschaft anerkannt, dennoch werden die meisten Menschen in Deutschland sie weiterhin als Sekte betrachten.

16 Dabei haben Experimente zu diesem Verhalten gezeigt, dass solche Reaktionen scheinbar unabhängig von spezifischen Inhalten einer „Wir“-Gruppenidentität funktionieren. So zeigen Tajfel und Turner (1986) z.B. in einem Experiment die spontane und kompetitiv ausgeprägte Gruppenbildung anhand unbedeutender Unterscheidungen (Vorliebe für bestimmte Künstler oder auch ganz ohne Merkmalsbildung der Gruppen). 
Gruppen (Intergruppenkontakt) tendenziell positiv auf deren Einstellungen zueinander auswirkt. Insofern erscheinen Dialoge zunächst als weichenstellende Möglichkeit der Konfliktvermeidung.

Darüber hinaus werden drei konkrete Ansätze zur Konfliktlösung in bestehenden Grenzdiskursen gesehen (vgl. Wagner 2000). Ein erster Lösungsansatz sieht vor, die Grenzen der Gruppenmitgliedschaft durch die Individualisierung der Beziehungen der Gruppenmitglieder zu überlagern. Dieser Ansatz erscheint erfolgreich bezüglich der Verbesserung der persönlichen Beziehung zueinander zu sein, allerdings bestehen starke Bedenken bezüglich des Effekts für eine Generalisierung dieser persönlichen Erfahrung auf die Gesamtgruppe (vgl. Hewstone und Klink 1994). So können etwa Christine und Peter nach solchen Erfahrungen zwar freundschaftliche Beziehungen zu Mehmet und Hatice haben, doch muss das keinen Effekt auf die gegenseitige Wahrnehmung von Muslim^innen oder Christ`innen als Gruppengesamtheiten haben: Vielmehr werden Mehmet und Hatice letztlich nicht mehr als typische Muslim*innen und Peter und Christine nicht mehr als typische Christ*innen wahrgenommen. Im interreligiösen Dialog ist dieses Phänomen durchaus gut bekannt, insbesondere in Dialoggruppen, in denen langjährige Beziehungen zueinander geführt werden, die teilweise bereits in Freundschaften übergehen, wenn dann gegenüber den außenstehenden, nur als traditionell-gläubig wahrgenommenen Muslim^innen oder Christ*innen oder auch den entsprechenden Gemeinden gegenüber Abwertungen vorgenommen werden. Hier verschiebt sich letztlich nur unbemerkt die Gruppengrenze: Die Dialog-/Freundschaftsgruppe wird zum identifikatorischen Bezug und setzt eine Grenze zu den Nicht-Gruppenmitgliedern in herkömmlich (negativ) orientalisierender Grammatik.

Zwei weitere Lösungsansätze betreffen direkt die Verschiebung der bestehenden Grenzen: Zum ersten die Kreuzkategorisierung, über die eine neue Kategorisierung eingezogen wird, die die bestehende abmildern soll (s.u. Grafik 2). So können Mehmet und Peter sowie Hatice und Christine neue Gruppen jenseits ihrer konfessionellen Zugehörigkeit zum Beispiel über ihre je gemeinsame Geschlechtsidentität bilden. Solche kreuzenden Grenzdiskurse werden von einigen Dialoggruppen umgesetzt, indem etwa Frauendialoggruppen gebildet werden, die nicht allein ihre unterschiedlichen konfessionellen religiösen Bezüge zum Thema machen, sondern insbesondere gemeinsam das feministische Ziel verfolgen, gegen geschlechterbezogene Ungerechtigkeiten in ihrer jeweils eigenen Konfession zu kämpfen und sich dabei gegenseitig zu unterstützen. Kreuzkategorisierungen können allerdings - so auch in der Sozialpsychologie bekannt - die Antagonismen zwischen den Gruppen verschärfen, insbesondere, wenn Personen mehreren negativen Kategorien anzugehören scheinen. Hier könnte das bedeuten, dass sich alle negativen Kategorisierungen dann auf den muslimischen Ma- 
cho oder den katholischen Patriarchen konzentrieren und damit diese Grenzmarkierung betonen und unter Umständen auch gegenseitig verstärken. ${ }^{17}$

Der dritte Lösungsansatz bezieht sich auf das Einziehen einer dritten übergeordneten Kategorisierung bzw. Grenze. Diese Lösungsstrategie darf dabei nicht als eine einseitige hierarchisierende Grammatik der Einschließung funktionieren, da sie sich nach Wagner nur umsetzen lasse, sofern alle Mitglieder darin ein gemeinsames Ziel verfolgen oder damit gegen einen gemeinsamen „Feind“ vorgehen. Im interreligiösen Dialog kann dies insbesondere in dem Versuch gesehen werden, die beteiligten Religionen als „abrahamitische Religionen“ zu verstehen und darüber Gemeinsamkeit herzustellen, wie oben bereits als Grammatik der Segmentierung beschrieben.

\section{Individualisierung}

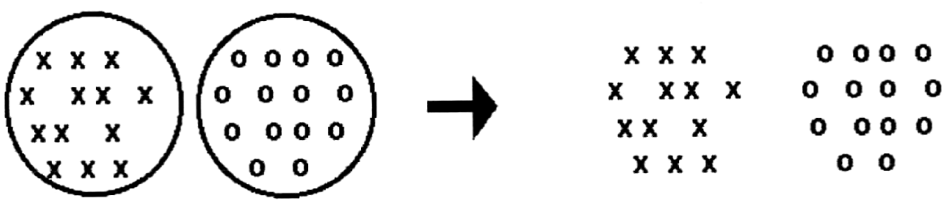

Kreuzkategorisienung

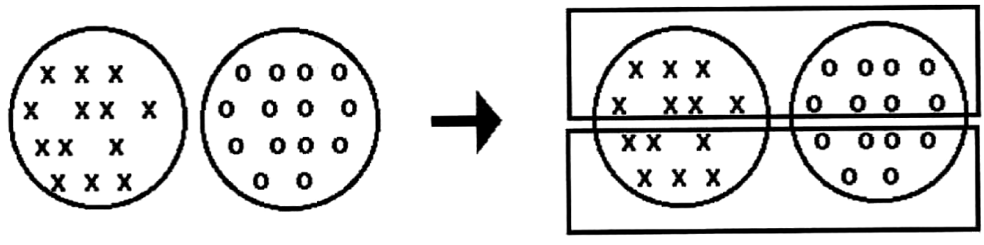

Übergeordnete Kategorisierung
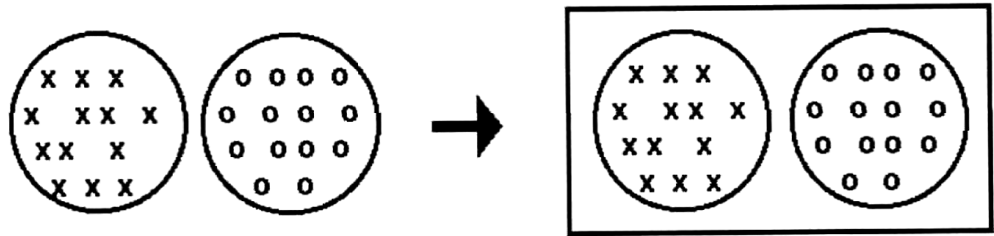

Grafik 2: Ulrich Wagner 2000, 37.

17 Vgl. hierzu auch Forschungen zur Intersektionalität (z. B. Klinger und Knapp 2005). 
Allerdings bedarf dieser letzte, wie alle anderen Ansätze auch, der gesellschaftlichen Unterstützung, das heißt, die Befürwortung der Intergruppenbeziehung durch gesellschaftliche Institutionen und Autoritäten ist eine der Bedingungen für ihr Gelingen (Pettigrew 2008). In der Sozialpsychologie gelten zudem drei weitere Bedingungen, die wichtig sind, um eine konfliktreduzierende Wirkung bei Interaktionen zwischen Gruppen erzeugen zu können, nämlich:

- „dass die Begegnungen als intime und nicht als formale Kontakte aufgefasst werden",

- „dass sie kooperativ gemeinsam übergeordnete Ziele verfolgen“ und

- „dass die Interaktionspartner zumindest in der gegebenen Interaktions-Situation den gleichen Status haben“ (Wagner 2000, 39).

Während die ersten beiden Bedingungen implizit auf die oben angesprochenen Grenzdiskurse (Individualisierung, übergeordnete Kategorisierung) Bezug nehmen, eröffnet die dritte Bedingung neue gesellschaftliche Zusammenhänge. Statusunterschiede können Unsicherheiten und Bedrohungsgefühle der Beteiligten fördern, wodurch die Neigung gegenseitiger Abwertung zunimmt. Im interreligiösen Dialog wird das Thema unter dem Stichwort „Augenhöhe“ diskutiert. Augenhöhe bzw. Status kann sich durch Verschiedenes repräsentieren. In den Gruppen kann sich dies etwa an der Verteilung von Ämtern und Moderationsaufgaben bemerkbar machen, aber auch am Bildungs- und Erwerbstatus der Teilnehmer ${ }^{\star}$ nen sowie der quantitativen Verteilung der beteiligten Gruppen. Bei den befragten Dialoginitiativen hat sich beispielsweise häufig die Tendenz gezeigt, die bestehenden ungleichen gesellschaftlichen Machtverhältnisse in der Dialoggruppe über Statusunterschiede auf den genannten Ebenen zumeist ungewollt zu reproduzieren.

\section{Religion in der Boundary Work des interreligiösen Dialogs}

Wie wirkt sich nun diese Grenzarbeit im interreligiösen Dialog auf das Religionsverständnis aus? Die Grenzmarkierungen und -verschiebungen im interreligiösen Dialog führen letztlich auch zu religionsproduktiven Aushandlungen innerhalb wie außerhalb des Dialoggeschehens.

Die einschließende Grammatik bedeutet für das Religionsverständnis letztlich nicht nur die Beibehaltung der bestehenden konfessionellen Grenzen, sondern gegebenenfalls ihre Überstülpung über die Anderen, um sie zu Eigenen zu machen. Religion bildet hierbei eine ganz eigene Sphäre der Wahrnehmung und 
Wirklichkeit, die durch Differenzerfahrung scheinbar nicht ins Wanken gerät. Neben Akteuren mit deutlich fundamentalisierenden religiösen Haltungen wie evangelikalen Christinnen, die letztlich jeden Dialog als eine Missionseinladung verstehen, oder solchen Musliminnen, die die Anderen unter ihre eigene konfessionelle Deutung der verfälschten „Religionen des Buches“ zusammenfassen, können auf diese Grammatik aber auch Personen mit betont offener religiöser Haltung zurückgreifen. Letztere verstehen dann Religion eher als spirituelle Haltung, die in allen Menschen zu finden sei - auch ohne deren Wissen. Erstere, „fundamentalisierende“ Haltungen sind im interreligiösen Dialog seltener anzutreffen.

Im Rahmen der Grammatik der Orientalisierung wird Religion für den Dialog als unterstützender (positiver) oder als hemmender (negativer) Impuls für eine Konfliktregulierung verstanden. Dabei wird der Diskurs über Religion und Religiosität im interreligiösen Dialog vielfach vom gesellschaftlichen und medial vermittelten Integrationsdiskurs überlagert. Es herrscht hier dann ein eher funktionales Verständnis von Religion und insbesondere der Auseinandersetzung über Religion vor. Mittels Religion wird etwa das Projekt der "Zivilisierung des Islam“ mit dem Ziel seiner Integration in die „moderne westliche Gesellschaft“ betrieben. Negative Impulse von Religion sollen hier so weit verändert werden, dass die jeweilige Religion allgemein eine unterstützende Funktion für die Integration des Einzelnen wie der Gesellschaft entfalten kann. Aber auch in positiv orientalisierender Wahrnehmung von Religion wird diese als Medium genutzt; nämlich für die Befriedigung eigener religiöser Bedürfnisse im interreligiösen Dialog, so, wenn neue, körperbezogene rituelle Formen des Ausdrucks gesucht und gemeinsam ausprobiert werden (z.B. sufische Spiritualität, Meditation, katholische Liturgie), wie auch, wenn intellektuelle sinnsuchende Auseinandersetzung mittels Religion in den Vordergrund des interreligiösen Dialogs rückt. Die Begegnung und Auseinandersetzung mit einer anderen Religion motiviert manche Teilnehmer ${ }^{\star}$ innen für eine neue oder manches Mal auch erstmalige Beschäftigung mit der eigenen Konfessionszugehörigkeit und/oder der eigenen Religiosität. Manche Teilnehmer ${ }^{\star}$ innen betonen, dass ihnen eine solche Auseinandersetzung in ihren Heimatgemeinden fehlt, andere wiederum berichten, dass sie sich nur aufgrund ihres Interesses für die „Fremden“ überhaupt mit der eigenen Religion und Religiosität auseinandersetzen.

Die segmentäre Grammatik der Grenzmarkierung zeigt ein gemeinsames, aber flexibles und entsprechend nur bedingt essentialistisches Religionsverständnis, das, wie dargelegt, entweder auf den einen Gott der abrahamitischen Traditionen setzt oder die Anerkennung von Transzendenz/Spiritualität überhaupt an die Spitze der Pyramide stellt. In den Dialogen selbst bedeutet dies nicht zwangsläufig, dass die konfessionellen Identitäten der Teilnehmer^innen sich verändern 
oder gar aufgeweicht werden. Vielmehr versteht man sich als vom gleichen Ursprung kommend und darum in Maßen in religiösen Dingen als verwandt. Die gemeinsame Identität ist allerdings kontextabhängig in ihrer Bedeutsamkeit. In der eigenen Gemeinde und für den persönlichen Glauben kann es unter Umständen von nur geringer bis keiner Bedeutung sein, dass man sich zur Familie der abrahamitischen Religionen zählt, auch wenn dies in der Begegnung und der grundsätzlichen Haltung zueinander im konkreten Dialogkontext wichtig ist.

Wenn allerdings die Wahrnehmung von einer übergeordneten Gemeinsamkeit so weit an Bedeutung gewinnt, dass die herkömmlichen konfessionellen Grenzen dadurch aufgeweicht werden oder sich im Austausch durch die Erfahrung miteinander und die Identifizierung mit dem neuen Dialogkollektiv verschieben, sind hier recht individuelle Umgangsweisen mit und Verständnisse von Religion anzutreffen; insbesondere, wenn die Gruppen dann eigene religiöse Rituale und intensive Freundschaftsgruppen bilden. Solch ein religiöser Identitätswandel im interreligiösen Austausch wird von Teilnehmer^innen allerdings meist nur sehr zurückhaltend zum Ausdruck gebracht, ${ }^{18}$ obwohl solche Entwicklungen im interreligiösen Dialog von zentralen Akteur`innen als grundlegend propagiert werden, da Offenheit für religiöse Veränderung als notwendig für die Möglichkeit von „echter“ Begegnung gesehen wird. ${ }^{19}$ Die Zurückhaltung im Konkreten mag auch damit zusammenhängen, dass seitens der Gemeinden bzw. deren Leitungen durchaus starke Skepsis gegenüber solcher Art Begegnungen geäußert wird, die auf der Graswurzelebene gepflegt werden. ${ }^{20}$ Das zeigt sich auch in der Verschärfung des Tons seitens der evangelischen Kirche gegenüber derartigen GrassrootsDialoggruppen in den letzten 10 Jahren. Es scheint so, als würde der Ton schärfer werden, je mehr sich solche unabhängigen Gruppen in Deutschland etablieren. ${ }^{21}$

18 Über den schwierigen Zugang zu solchen Wandlungsprozessen und die Frage, was sie begünstigt bzw. worin sie begründet liegen vgl. Klinkhammer und Neumaier (im Druck).

19 Vgl. hierzu auch Programmschriften zum interreligiösen Dialog wie die bereits erwähnten von Swidler 1987, 2014 u.a.

20 Der Entwurf eigener offizieller Projekte des Dialogs seitens der Kirchen und der islamischen Dachverbände kann sowohl als Unterstützung als aber auch als Zivilisierungs- oder GovernanceStrategie gegen ein unkontrolliertes Ausufern der Grassroots-Dialoge verstanden werden. Siehe z.B. das Projekt der ACK zusammen mit DITIB „Weißt du, wer ich bin?“, das dann auch seitens der ACK Materialien erstellt hat, die im Dialog der Grassroots-Gruppen zur Nutzung kommen sollen. Die DITIB hat in eigens eingerichteten Kursen „Dialogführer^innen“ ausgebildet. Hierbei handelt es sich vornehmlich um zukünftige Moscheeführer^innen. Der Dialogos-Studie wurde damals seitens der DITIB der Zugang zu diesen Kursen und den Kursteilnehmer^innen allerdings verwehrt. 21 Dazu gehört sicherlich auch die Wahrnehmung einer zunehmenden Etablierung der islamischen Dachverbände in Deutschland, die allerdings durch die zunehmende Entdemokratisierung der Türkei einen bedeutenden Rückschlag erhalten hat. 
So sah sich die EKD 2006 - auf einem bisherigen Höhepunkt der Entstehung der freien interreligiösen Dialoginitiativen ${ }^{22}$ - offenbar gefordert, $z u$ ihrer letzten, eher Grenzen öffnenden Handreichung (2000) zum christlich-muslimischen Dia$\log$ nun eine neue, konfessionelle Grenzen betonende Handreichung für den Dia$\log$ „Klarheit und gute Nachbarschaft“ herauszugeben. Diese neue Veröffentlichung widmet sich nicht mehr der Frage, wie eine vertrauensvolle Öffnung gegenüber dem Anderen theologisch und praktisch möglich ist, sondern macht konfessionelle Identität stark und betont den missionarischen und konkurrierenden Charakter beider Religionen, dem in der Begegnung letztlich durch klare Grenzziehung Rechnung getragen werden müsse. Dialog ist danach nunmehr vor allem eine Aufgabe in den „Spannungsbereichen gesellschaftlicher Integration und des praktischen Zusammenlebens“, zu der es „keine zukunftsträchtige Alternative" gebe (Evangelische Kirche Deutschland 2006, 14) und nicht mehr der Wunsch, Musliminnen in „Offenheit zu begegnen“ und „Verständigung“ sowie Selbstverständigung über den eigenen Glauben im Dialog zu suchen (Evangelische Kirche Deutschland 2000, 11-15). Im Dialog müsse demgegenüber heute jeder „Anschein der Religionsvermischung“ vermieden werden (Evangelische Kirche Deutschland 2006, 118); so sei darum z.B. jedes gemeinsame Gebet „zuverlässig zu vermeiden“ (Evangelische Kirche Deutschland 2006, 117), während in der Handreichung von 2000 noch das Wirken und der Nutzen der „Grenzgänger“ im Dialog ${ }^{23}$ positiv betont und das gemeinsame Gebet als Möglichkeit und bestehende Praxis im Dialog (vorsichtig) anerkannt wird (Evangelische Kirche Deutschland 2000, 41-45).

In den Gruppen des interreligiösen Dialogs wurde die neue Handreichung äußerst kritisch diskutiert (vgl. Micksch 2007) und als großes Hemmnis für den interreligiösen Dialog bewertet. Spätestens seitdem bilden Kirchen wie islamische Dachverbände in vielen Grassroots-Gruppen den dritten ausgeschlossenen Part der Grenzziehung im interreligiösen Dialog. ${ }^{24}$

22 Dieser fällt in etwa in das Ende des BMFSJ-Programms „entimon“, das nicht nur viele interreligiöse Initiativen unabhängig von deren Anbindung an Kirchen oder Dachverbänden gefördert hat, sondern durch sein Programm auch für deren Entstehung insgesamt geworben hat.

23 So heißt es in der Handreichung von 2000: „Obwohl sich der innerchristliche Dialog auf einer prinzipiell anderen Ebene vollzieht als interreligiöse Gespräche, brauchen wir auch in der Begegnung mit dem Islam heute solche Grenzgänger zwischen den Kulturen und Religionen. Wir brauchen die teilnehmende Erfahrung von Menschen, die durch eine religionsverschiedene Ehe oder aus dem Dialog des Lebens heraus in einer doppelten Heimat leben und uns durch ihre Erfahrungen helfen können.“ (Evangelische Kirche Deutschland 2000, 40).

24 Die katholische Kirche hat zwar z.T. sehr engagierte Theolog*innen in leitenden Rollen einiger institutionalisierter interreligiöser Dialoge, allerdings ist die dogmatische Auffassung nicht erst seit der Erklärung Dominus Jesus (2000) deutlich inklusivistisch abgesteckt. In manchen der Grass- 
Die Deutungshoheit zur Grenzziehung zwischen Orthodoxie und Heterodoxie möchte man sich in den Dialoggruppen gerade nicht aus der Hand nehmen lassen. Die Nichtakzeptanz des interreligiösen Dialogs durch das religiöse bzw. institutionelle Establishment der am Dialog beteiligten Religionsgemeinschaften mag allerdings dennoch Folgen haben, da eine der Bedingungen für die befriedende Wirkung der Intergruppenbeziehung dann nicht gegeben ist, nämlich die oben genannte Befürwortung durch gesellschaftliche Institutionen oder Autoritäten. Das heißt auch, dass die orientalisierenden und segmentären Grammatiken der Grenzziehung im interreligiösen Dialog für eine positiv integrierende Wirkung einer öffentlichen Akzeptanz bedürfen, ansonsten schaffen sie vor allem neue Grenzen und Fronten zwischen den etablierten Religionen und den interreligiösen Grassroots-Gruppen. Die Verhandlung von Religion verläuft also nicht nur auf verschiedenen Weisen im interreligiösen Dialog selbst - wie der Durchgang durch die drei Grammatiken gezeigt hat -, sondern immer auch in Bezug zu den gesellschaftlichen und religionspolitischen Rahmenbedingungen. Angesichts dessen ist schließlich zu konstatieren, dass das Zusammenleben durch interreligiösen Dialog allein nicht friedlicher wird. Religion als zentrales Konstrukt, an dem die Grenzbearbeitung entlangführt, erscheint eher als umkämpfte Ressource; und zwar auch im Dialog selbst, wenn es z.B. um die Zivilisierung der einen und die Hegemonie der anderen geht. Auch eine alles umschließende Begrifflichkeit wie die der „abrahamitischen Religion“ oder die individualisierte Grenzüberschreitung persönlicher Religiosität/Spiritualität vermögen kaum zu einer Etablierung friedlicher Koexistenz führen, wenn sie die identitäre Verteidigung der Grenzen durch die übergeordneten Institutionen der Kirchen und islamischen Verbände gegenüber den Dialoggruppen hervorrufen.

Die Ambivalenzen, die sich in den Beobachtungen zu Grenzziehungen und Grenzverschiebungen im interreligiösen Dialog zeigen, sind letztlich eben auch der grundsätzlich paradoxen Struktur des interreligiösen Dialogs geschuldet: Mit der sich im interreligiösen Dialog vollziehenden Konzentration auf die unterschiedlichen religiösen Identitäten wird immer eine imaginäre Grenze zwischen Konfessionen - die es zunächst zu definieren und dann immer wieder zu überschreiten gilt - aufrechterhalten. Die Frage, ob der christlich-muslimische und der interreligiöse Dialog überhaupt förderlich für das Aufbrechen von Grenzen ist, ist darum nicht trivial - zumindest solange nicht, wie institutionalisierte konfes-

roots-Dialoggruppen ist es bei allzu großer Abweichung davon durchaus auch schon zum Abzug einer Vertreter*in aus der Dialoggruppe seitens der Kirchenleitung gekommen; andere berichten, dass sie sich gegenüber ihren kirchlichen Vorgesetzten und/oder Gemeinden nicht frei fühlen, den Dialog so offen zu führen, wie sie es persönlich für richtig halten. 
sionelle Strukturen die Rahmenbedingungen für das Zusammentreffen und die Auseinandersetzung bilden.

\section{Zum Umgang der Religionswissenschaft mit Grenzziehungsdynamiken im interreligiösen Dialog}

Nachdem im Vorangegangenen die konkreten Ausformungen struktureller Grammatiken und sozialpsychologischer Dynamiken der Grenzziehung im interreligiösen Dialog auf der Grundlage der Ergebnisse der Dialogos-Studie aufgezeigt wurden, lässt sich nun auch die Involvierung von Religionswissenschaft in solche Grenzziehungsdynamiken diskutieren. Ein Engagement der Religionswissenschaft für den interreligiösen Dialog, wie es beispielsweise Wilfred Cantwell Smith (1962) gefordert hat und heute immer noch von der Religionsphänomenologie nahestehenden Religionswissenschaftler ${ }^{\star}$ innen gefordert wird, muss vor diesem Hintergrund kritisch hinterfragt werden. ${ }^{25}$ Es lässt sich aus dem Vorangegangenen vielmehr folgern, dass interreligiöse Aktivitäten selbst höchst religionsproduktiv sind und damit in erster Linie ebenso zum religionswissenschaftlichen Untersuchungsgegenstand gehören wie die Untersuchung traditionsreicher religiöser Kollektive (z.B. der christlichen Kirchen) oder solcher jüngeren Datums (z.B. der islamischen Verbände). Zwar haben die interreligiösen Dialoge bislang weder quantitativ noch qualitativ so weit Bedeutung erlangt, dass sie die herkömmlichen institutionalisierten Konfessionsgrenzen ins Wanken geraten lassen - was, wie ich dargelegt habe, in der Regel auch kaum Ziel der Teilnehmer*innen am Dialog ist. Allerdings werden diese quasi-institutionalisierten Grassroots-Dialoge von zahlreichen Auseinandersetzungen auch intrakonfessioneller Artflankiert, ${ }^{26}$ die ebenso wie der interreligiöse Dialog zeigen, dass sich die religiöse Gegenwartskultur längst durch eine tiefgreifende Differenzierung und Individualisierung religiöser Grenzmarkierung auszeichnet, so dass klare kollektiv-konfessionelle Zuordnungen für einzelne Akteure im Dialog mitunter mehr als schwierig erscheinen. Gleichzeitig zeigte sich, dass sich

25 Ich möchte hiermit nicht die Berufsmöglichkeiten für Religionswissenschaftler^innen außerhalb der Wissenschaft einschränken, sondern betonen, dass Dialog-Moderation weder eine wissenschaftliche Tätigkeit ist, noch inhärent zu den Zielen von Religionswissenschaft gehört - gleichwohl sie von Religionswissenschaftler^innen u.U. sicherlich sehr kompetent durchgeführt werden kann.

26 Z.B. durch Auseinandersetzungen im Netz; vgl. z.B. Anna Neumaiers Analyse katholischer Onlinediskurse (2016). 
im interreligiösen Dialog konfessionelle Grenzziehungen unter Umständen verstärken und/oder neue quasi-konfessionelle Identitäten (abrahamitische Religionen, Monotheismus u.a.) geschaffen werden.

In dieser Situation ist es die Aufgabe der Religionswissenschaft, aufmerksam für diversifizierte Praktiken innerhalb des interreligiösen Dialogs zu sein, um die differenzierte bekenntnisbezogene Grenzarbeit sowie deren religionsdynamische Wirkung in den Blick nehmen zu können. Neben einer vertieften Untersuchung des Einflusses des interreligiösen Dialogs auf die religiöse Identität des Einzelnen ist auch die Dynamik der Pluralisierung bzw. Diversifizierung von Religion weiterhin unter anderem als Folge von Religionskontakten zu untersuchen. Wie das oben angeführte Beispiel der Reaktion der EKD auf die Dialoge zeigt, ist neben einem individualisierten Umgang auch ein fundamentalisierender, abgrenzender Umgang des religiösen Establishments gegenüber der frei zugänglichen Vielfalt und damit eine Revitalisierung der Konfessionalisierung nicht ausgeschlossen. Allerdings ist aufgrund einer zugleich zunehmenden Individualisierung wohl zu erwarten, dass dadurch eher die Diversität der institutionell abgesicherten konfessionellen Grenzen im (inter-)religiösen Diskurs steigen wird. Der Dialog hält jedenfalls weder das eine noch das andere auf; im Gegenteil fördert er einerseits individualisierte Prozesse von Religionsaneignung und evoziert gleichzeitig die Verteidigung angestammter institutionalisierter und neuer konfessioneller Identitäten.

\section{Literatur}

Allport, Gordon. 1954. The Nature of Prejudice. Reading, MA: Addison-Wesley.

Barth, Fredrik. 1969. Ethnic Groups and Boundaries. The Social Organization of Culture Difference. Bergen: Universitetsforl.

Baumann, Gerd. 2004. "Grammars of Identity/Alterity. A Structural Approach.” In Grammars of Identity/Alterity. A Structural Approach hg. v. G. Baumann, A. Gingrich, 18-50. New York, Oxford: Berghahn Books.

Bertelsmann Stiftung, Hg. Religionsmonitor 2008. Muslimische Religiosität. Überblick zu religiösen Einstellungen und Praktiken. Gütersloh: Gütersloher Verlagshaus.

Braunwarth, Esther. 2011. Interkulturelle Kooperation in Deutschland am Beispiel der Gesellschaften für christlich-jüdische Zusammenarbeit. München: Herbert Utz Verlag.

Brunotte, Ulrike. 2009. „Religion und Kolonialismus.“ In Europäische Religionsgeschichte. Ein mehrfacher Pluralismus, Bd. 1., hg. v. Hans G. Kippenberg, Jörg Rüpke und Kocku v. Stuckrad, 339-369. Göttingen: Vandenhoeck \& Ruprecht.

Buber, Martin. [1923] 1965. Das dialogische Prinzip. Heidelberg: Schneider.

Council of Europe. 2008. White Paper on Intercultural Dialogue. Straßburg.

Evangelische Kirche Deutschland. 2000. Zusammenleben mit Muslimen in Deutschland. Gestaltung der christlichen Begegnung mit Muslimen. Eine Handreichung des Rates der EKD. Hannover. 
Evangelische Kirche Deutschland. 2006. Klarheit und gute Nachbarschaft. Christen und Muslime in Deutschland. Eine Handreichung des Rates der EKD. Hannover.

Forst, Rainer. 2003. Toleranz im Konflikt. Frankfurt am Main: Suhrkamp.

Gadamer, Hans-Georg. 1960. Wahrheit und Methode. Grundzüge einer philosophischen Hermeneutik. Tübingen: Mohr.

Hewstone, Miles und Andreas Klink. 1994. „Intergruppenattribution.“ In Attributionstheorie, hg. v. Friedrich Försterling und Joachim Stiensmeier-Pelster, 73-104. Göttingen: Hogrefe.

Klinger, Cornelia und Gudrun-Axeli Knapp. 2005. „Achsen der Ungleichheit - Achsen der Differenz. Verhältnisbestimmungen von Klasse, Geschlecht, ,Rasse“/Ethnizität.“ Transit - Europäische Revue 29:72-96.

Klinkhammer, Gritt. 2009. "The Emergence of Transethnic Sufism in Germany: From Mysticism to Authenticity." In Sufis in Western Societies. Global Networking and Locality, hg. v. Markus Dreßler, Ron Geaves und Gritt Klinkhammer, 130-147. London: Routledge.

Klinkhammer, Gritt, Hans-Ludwig Frese, Ayla Satilmis und Tina Seibert. 2011. Interreligiöse und interkulturelle Dialoge mit MuslimInnen in Deutschland: eine quantitative und qualitative Studie. Universität Bremen und http://elib.suub.uni-bremen.de/edocs/00102006-1.pdf (zuletzt abgerufen am 28.01.2019).

Klinkhammer, Gritt und Anna Neumaier. Im Druck. “Narrating Stability within Interreligious Dialogue. First Results of a Qualitative Inquiry on Consequences of Plurality Experiences for Religious Identity." In Religious Pluralisation. A Challenge for Modern Societies, hg. v. Wolfram Weiße und Anna Körs, 20 Seiten. Hamburg: Springer.

Kongregation für die Glaubenslehre, Hg. 2000. Erklärung Dominus Jesus. Über die Einzigkeit und die Heilsuniversalität Jesu Christi und der Kirche. Rom.

Micksch, Jürgen, Hg. 2007. Evangelisch aus fundamentalem Grund. Wie sich die EKD gegen den Islam profiliert. Frankfurt am Main: Otto Lembeck.

Micksch, Jürgen und Rita Süssmuth. 2005. Islamforen in Deutschland. Frankfurt am Main: Lembeck.

Neumaier, Anna. 2016. religion@home? Religionsbezogene Online-Plattformen und ihre Nutzung. Eine Untersuchung zu neuen Formen gegenwärtiger Religiosität. Würzburg: Ergon.

Pettigrew, Thomas F. 2008. „Future Directions for Intergroup Contact Theory and Research.“ International Journal of Intercultural Relations 32:187-199.

Said, Edward. [1978] 2009. Orientalismus. Frankfurt am Main: S. Fischer.

Smith, Cantwell. 1962. The Meaning and End of Religion. New York: Macmillan.

Sperber, Jutta. 1999. Dialog mit dem Islam. Göttingen: Vandenhoeck \& Ruprecht.

Swidler, Leonard. 1987. Towards a Universal Theology of Religion. Maryknoll/New York: Orbis Books.

Swidler, Leonard. 2014. Dialogue for Interreligious Understanding. Strategies for the Transformation of Culture-Shaping Institutions. New York: Palgrave Macmillan.

Tajfel, Henri und John C. Turner. 1986. "The Social Identity Theory of Intergroup Behaviour." In Psychology of Intergroup Behaviour, hg. v. Stephen Worchel und William G. Austin, 7-24. Chicago: Nelson Hall.

Wagner, Ulrich. 2000. Gruppenprozesse: Intergruppenprozesse. Fernuniversität Hagen, www.uni-marburg.de/fb04/team-sozialpsychologie/lehre/downloads/download\% 20hagenpapier.pdf (zuletzt abgerufen am 9.11.2017). 